\title{
Angle-selective perfect absorption with two-dimensional materials
}

\author{
Linxiao Zhu' ${ }^{1}$ Fengyuan $\mathrm{Liu}^{2}$, Hongtao $\mathrm{Lin}^{3}$, Juejun $\mathrm{Hu}^{3}$, Zongfu $\mathrm{Yu}^{4}$, Xinran Wang ${ }^{2}$ and Shanhui Fan ${ }^{5}$
}

Two-dimensional (2D) materials have great potential in photonic and optoelectronic devices. However, the relatively weak light absorption in 2D materials hinders their application in practical devices. Here, we propose a general approach to achieve angleselective perfect light absorption in 2D materials. As a demonstration of the concept, we experimentally show giant light absorption by placing large-area single-layer graphene on a structure consisting of a chalcogenide layer atop a mirror and achieving a total absorption of $77.6 \%$ in the mid-infrared wavelength range $(\sim 13 \mu \mathrm{m})$, where the graphene contributes a record-high $47.2 \%$ absorptivity of mid-infrared light. Construction of such an angle-selective thin optical element is important for solar and thermal energy harvesting, photo-detection and sensing applications. Our study points to a new opportunity to combine 2D materials with photonic structures to enable novel device applications.

Light: Science \& Applications (2016) 5, e16052; doi:10.1038/lsa.2016.52; published online 25 March 2016

Keywords: angle-selective perfect absorption; angle-selective thin optical element; graphene; mid-infrared; two-dimensional materials

\section{INTRODUCTION}

Two-dimensional (2D) materials such as graphene and transitionmetal dichalcogenide have generated significant recent interest in photonics and optoelectronics owing to their unique properties for light-matter interactions ${ }^{1-5}$. To exploit these properties for device applications, significant recent efforts have focused on combining these 2D materials with photonic structures, such as dielectric waveguides $^{2,6-13}$, plasmonic structures ${ }^{3,14-17}$ or photonic crystals ${ }^{18-27}$. Such combinations of 2D materials with photonic structures have led to the demonstration of near-complete absorption in single-layer graphene $^{22,23,27}$, as well as modulators ${ }^{6,7,11-13,25}$, detectors $^{8-10,28-33}$ and lasers $^{24}$

In this study, we consider a particularly simple geometry of a photonic structure incorporating a $2 \mathrm{D}$ material. The geometry, shown in Figure 1a, consists of a single layer of $2 \mathrm{D}$ material separated from a mirror by a dielectric spacer layer. This geometry has been previously used to enhance the absorption in a graphene layer of normal incident light ${ }^{34}$, but the demonstrated enhancement is rather limited. Here, we theoretically prove that such a structure provides a universal mechanism for achieving perfect absorption in the $2 \mathrm{D}$ material. In this geometry, for any standard 2D material in the wavelength range, in which the material has some loss, there always exists an angle of incidence, at which the absorption of the $2 \mathrm{D}$ material reaches unity. Therefore, the simple geometry in Figure la can function as an angleselective perfect absorber. Experimentally, we have shown that the geometry in Figure la can lead to a total absorption exceeding $77 \%$ in the mid-infrared wavelength range, in which the absorption in singlelayer graphene exceeds $47 \%$.

The construction of an angle-selective thin optical element has been of emerging interest recently owing to its importance for solar and thermal energy harvesting ${ }^{35-37}$ as well as photodetection ${ }^{38}$. Thus, our work points to a new opportunity of combining $2 \mathrm{D}$ materials with photonic structures to enable novel device applications. The demonstrated angle-selective perfect absorption in the infrared wavelength range alone can also be important for sensing applications ${ }^{17}$.

\section{MATERIALS AND METHODS}

\section{Fabrication of the structure}

The bare structure is deposited atop a polished silicon wafer. A 200-nm-thick chromium adhesion layer is first deposited using electron beam evaporation, followed by $200 \mathrm{~nm}$ of gold. After cleaning the surface of the $\mathrm{Au} / \mathrm{Cr} / \mathrm{Si}$ substrate, a $\mathrm{Ge}_{23} \mathrm{Sb}_{7} \mathrm{~S}_{70}$ chalcogenide layer $\sim 1.9 \mu \mathrm{m}$ thick is deposited on top using standard single-source thermal evaporation.

A single layer of graphene is grown by chemical vapor deposition on copper foil and transferred onto the structure with the standard Poly(methyl methacrylate) (PMMA) process ${ }^{39}$. After baking at $120^{\circ} \mathrm{C}$ for $5 \mathrm{~min}$, the PMMA is dissolved by soaking the sample in acetone for $6 \mathrm{~h}$ and acetic acid for $3 \mathrm{~h}$. Chemical doping ${ }^{40}$ is then performed by applying nitric acid vapor on the graphene layer. We control the doping of the graphene layer by varying the duration of nitric acid vapor treatment. Two samples were fabricated with 5min doping time and 40-s doping time, respectively.

${ }^{1}$ Department of Applied Physics, Stanford University, Stanford, CA 94305, USA; ${ }^{2}$ National Laboratory of Solid State Microstructures, School of Electronic Science and Engineering and Collaborative Innovation Center of Advanced Microstructures, Nanjing University, Nanjing 210093, China; ${ }^{3}$ Department of Materials Science and Engineering, Massachusetts Institute of Technology, MA 02139, USA; ${ }^{4}$ Department of Electrical and Computer Engineering, University of Wisconsin-Madison, Madison, WI 53706, USA and ${ }^{5}$ Department of Electrical Engineering, Ginzton Laboratory, Stanford University, Stanford, CA 94305, USA

Correspondence: ZF Yu, Email: zyu54@wisc.edu; XR Wang, Email: xrwang@nju.edu.cn; SH Fan, Email: shanhui@stanford.edu

Received 28 October 2015; revised 24 November 2015; accepted 26 November 2015; accepted article preview online 2 December 2015 

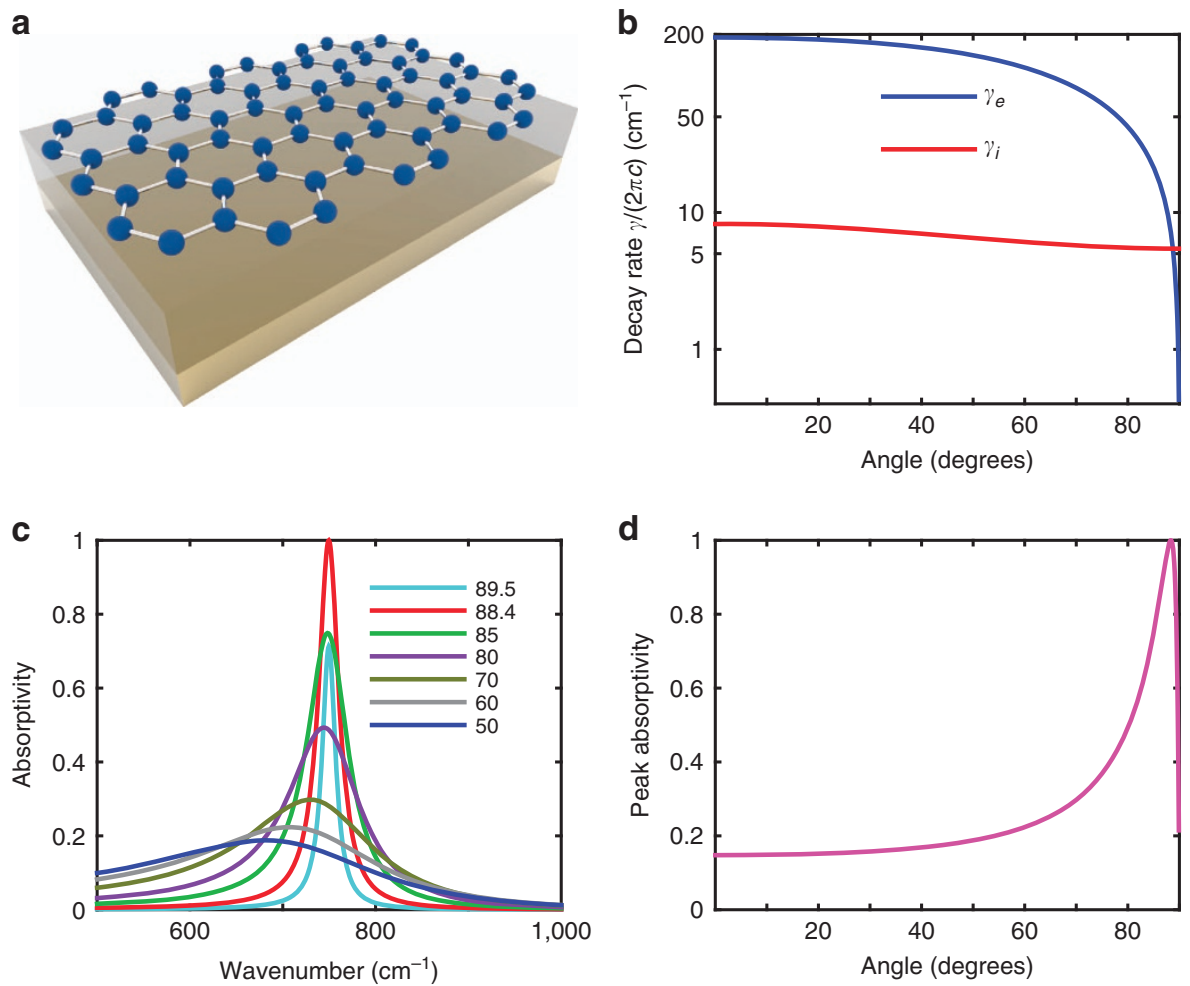

Figure 1 Decay rates and critical coupling. (a) Schematic of a structure consisting of a 2D material, separated from a mirror (light gray) by a dielectric spacer layer (dark gray). As an example, the 2D material is a single-layer graphene. (b) External and internal decay rates of the structure as a function of angle of incidence. The external decay rate ( $\gamma_{e}$, blue line) and internal decay rate ( $\gamma_{i}$, red line) are evaluated from Equations (7) and (8), respectively. (c) Calculated mid-infrared absorptivity of the structure, for varying angles of incidence. (d) Calculated peak absorptivity as a function of angle of incidence. In b, c and d, the structure consists of single-layer graphene separated from a PEC layer by a 1.9- $\mu$ m-thick dielectric layer with a refractive index of 2.1. The graphene is assumed to have a Fermi energy of $-500 \mathrm{meV}$ with a mobility of $750 \mathrm{~cm}^{2} \mathrm{~V}^{-1} \mathrm{~s}^{-1}$. In $\mathbf{b}, \mathbf{c}$ and d, s-polarization is considered. PEC, perfect electric conductor.

\section{Characterization of the structure}

At infrared wavelengths from $400 \mathrm{~cm}^{-1}$ to $2500 \mathrm{~cm}^{-1}$, a Fourier transform infrared spectrometer (Nicolet 6700, Thermo Fisher Scientific, Waltham, MA, USA) is used to characterize the reflectance of the samples, with a gold film used as a reflectance standard, as shown in Figure 2c-2e. Because the structure is planar, from the measured reflectance $R$, absorptivity $A$ can be calculated by $A=1-R$. A variable-angle reflection accessory (Seagull, Harrick Scientific, Pleasantville, NY, USA) equipped with KRS-5 substrate-based wire grid polarizer (Seagull FTIR polarizer, Harrick Scientific) allows for specular reflectance measurement at varying angles of incidence. The measurement sampling step in wave number is $1.9 \mathrm{~cm}^{-1}$. Nitrogen purging is performed for the FTIR and the variable-angle reflection accessory during the measurement.

Raman measurement of samples is performed using a laser with a 514-nm wavelength, which is the same wavelength of the laser used in Ref. 41. The Raman measurement in both doping cases is performed at five randomly selected locations on each structure.

\section{Extraction of absorption in single-layer graphene}

The external decay rate $\gamma_{e}$ results from the resonance mode of the bare structure. The internal decay rate results both from material loss inside the single-layer graphene $\gamma_{i, G}$ and parasitic loss $\gamma_{i, P}$ inside the metal and spacer layers. Using the coupled mode theory ${ }^{42,43}$, the absorptivity of the bare structure is:

$$
\alpha_{\text {Bare }}=\frac{4 \gamma_{e} \gamma_{i, P}}{\left(\omega-\omega_{0}\right)^{2}+\left(\gamma_{e}+\gamma_{i, P}\right)^{2}}
$$

and the absorptivity of the structure with graphene is:

$$
\alpha_{\text {Hybrid }}=\frac{4 \gamma_{e}\left(\gamma_{i, P}+\gamma_{i, G}\right)}{\left(\omega-\omega_{0}\right)^{2}+\left(\gamma_{e}+\gamma_{i, P}+\gamma_{i, G}\right)^{2}}
$$

Here, we have assumed that the resonance frequency $\omega_{0}$ remains unchanged with the introduction of the graphene layer. From Equations (1) and (2), by comparing the measured absorptivities of the structures with and without graphene, decay rates can be obtained, and the absorption in the graphene layer and the remaining structure can be extracted. The absorption in graphene is:

$$
\alpha_{G, \text { Hybrid }}=\frac{4 \gamma_{e} \gamma_{i, G}}{\left(\omega-\omega_{0}\right)^{2}+\left(\gamma_{e}+\gamma_{i, P}+\gamma_{i, G}\right)^{2}}
$$

and the parasitic absorption is:

$$
\alpha_{P, \text { Hybrid }}=\frac{4 \gamma_{e} \gamma_{i, P}}{\left(\omega-\omega_{0}\right)^{2}+\left(\gamma_{e}+\gamma_{i, P}+\gamma_{i, G}\right)^{2}}
$$

\section{Graphene conductivity in intraband transition regime}

The conductivity of doped graphene in the intraband regime ${ }^{44}$ can be described as:

$$
\sigma_{G}=\frac{-i D}{\pi(\omega-i \Gamma)}
$$

where the Drude weight, $D=\frac{e^{2}}{\hbar} v_{F} \sqrt{\pi n}$ and the relaxation rate, $\Gamma=\frac{e v_{F}}{h \mu_{G} \sqrt{\pi}}$. Here, Fermi velocity ${ }^{45}, \quad v_{F} \approx 10^{6} \mathrm{~ms}^{-1}$, carrier density, $n$ is related to Fermi energy, $E_{F}$ by $n=\frac{1}{\pi}\left(\frac{\left|E_{F}\right|}{h_{F}}\right)^{2}$, and $\mu_{G}$ is the carrier mobility. In the intraband transition regime, for s-polarization, the internal decay rate of graphene can then be expressed as:

$$
\gamma_{i}=\frac{c}{d} \cdot \frac{1}{\varepsilon} \cdot \frac{4 \alpha}{\frac{\hbar}{e v_{F}^{2}} \omega^{2} \mu_{G}+\hbar e v_{F}^{2} \frac{1}{\left|E_{F}\right|^{2} \mu_{G}}}
$$


a

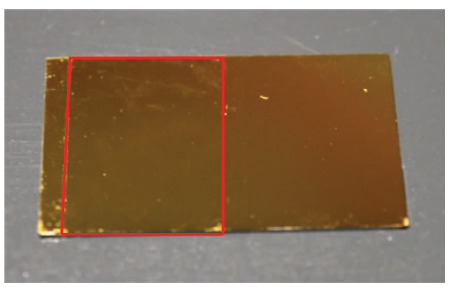

b

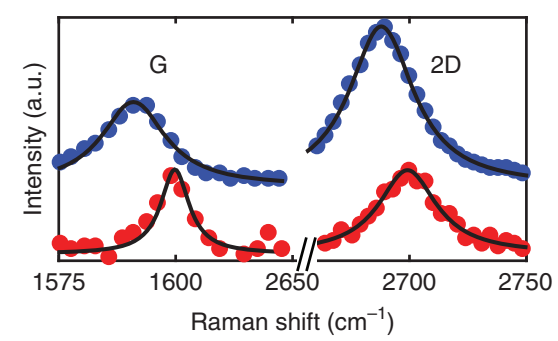

f

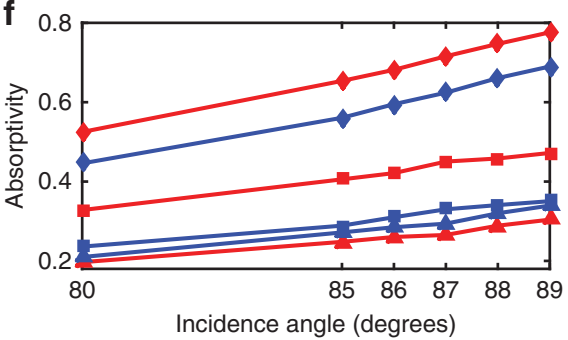

C

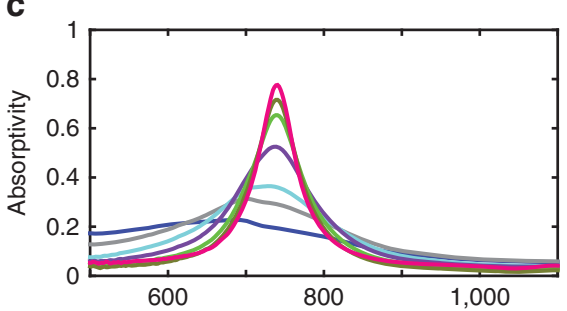

d
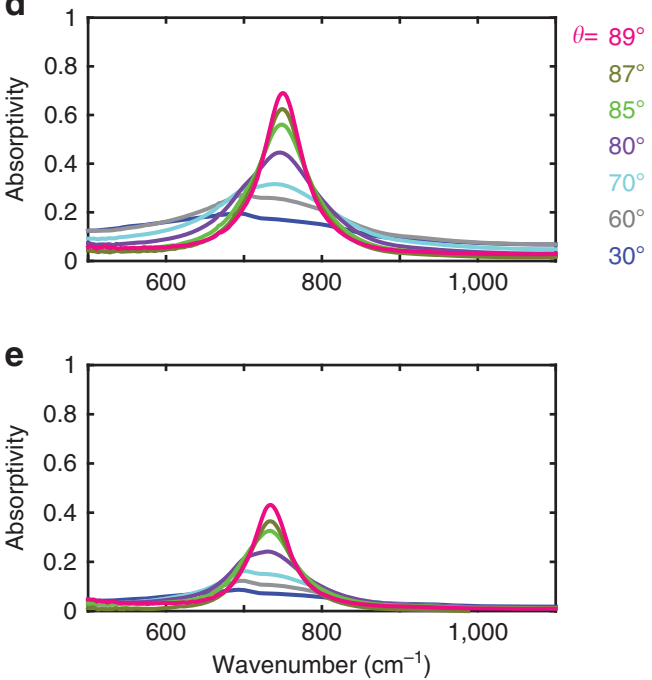

Figure 2 Device and experimentally measured absorptivity. (a) Photo of the structure. It consists of a doped single layer of graphene separated from a gold reflector by a 1.9- $\mu \mathrm{m}$-thick $\mathrm{Ge}_{23} \mathrm{Sb}_{7} \mathrm{~S}_{70} \mathrm{ChG}$ layer. Here, the red rectangle denotes the region that is coated with graphene. (b) Raman spectra for two structures with different graphene layers with different doping levels. The experimental spectra are shown as dots, and Lorentzian fittings are shown as black lines. The Raman spectra are vertically displaced for clarity. The graphene with 5-min (red dots) and 40-s (blue dots) doping durations are estimated to have Fermi energy $E_{F}$ of $-500 \mathrm{meV}$ and $-300 \mathrm{meV}$, respectively (see the Supplementary Information). (c-e) Measured angle-resolved absorptivity for structures. (c) The case of graphene at $E_{F}=-500 \mathrm{meV}$. (d) The case of graphene at $E_{F}=-300 \mathrm{meV}$. (e) The case of a bare structure without graphene. (f) Peak absorptivities for the whole structure (diamonds), the absorption inside graphene (squares) and parasitic absorption (triangles). Red and blue lines denote graphene with $E_{F}=-500 \mathrm{meV}$ and $E_{F}=-300 \mathrm{meV}$, respectively. $\mathbf{c}-\mathbf{f}$ are for s-polarization.

where $\alpha$ is the fine structure constant. We observe that the internal decay rate increases with increasing Fermi energy, $\left|E_{F}\right|$. For a fixed Fermi energy and resonance frequency, as a function of mobility, the maximal internal decay rate is $\frac{c}{d} \cdot \frac{1}{\varepsilon} \cdot \frac{2 \alpha\left|E_{F}\right|}{\hbar \omega}$ when $\mu_{G}=\frac{e v_{F}^{2}}{\omega \mid E_{F}}$.

\section{RESULTS AND DISCUSSION}

We consider the theoretical condition for perfect light absorption in the $2 \mathrm{D}$ material with the structure shown in Figure 1a. For simplicity, we assume that all layers except the $2 \mathrm{D}$ material is lossless and that the bottom mirror has $100 \%$ reflectivity. Such a structure supports a resonance with light trapped in the dielectric layer owing to the reflections at its top and bottom surfaces. The resonance mode amplitude can decay either through external radiation through the top surface, as characterized by an external decay rate $\gamma_{e}$, or by the absorption in the 2D material, as characterized by an internal decay rate $\gamma_{i}$. Complete absorption in the $2 \mathrm{D}$ material layer can be reached when the critical coupling condition $\gamma_{e}=\gamma_{i}$ is satisfied.

We consider first the $s$-polarization, whose electric field is perpendicular to the plane of incidence. The external decay rate $\gamma_{e}$ of the resonance mode can be derived as (see the Supplementary Information for a detailed derivation):

$$
\gamma_{e}=\frac{c}{d} \cdot \frac{\cos \theta}{\varepsilon-\sin ^{2} \theta}
$$

where $c$ is the velocity of light, $d$ is the thickness of the spacer layer, $\varepsilon$ is the relative permittivity of the spacer layer, and $\theta$ is the angle of incidence. The internal decay rate due to the absorption in the $2 \mathrm{D}$ material can be derived as (see the Supplementary Information):

$$
\gamma_{i}=\frac{c}{d} \cdot \frac{1}{\varepsilon} \cdot \operatorname{Re}(\sigma) Z_{0}
$$

where $\sigma$ is the $2 \mathrm{D}$ conductivity of the $2 \mathrm{D}$ material, and $Z_{0}=\sqrt{\mu_{0} / \varepsilon_{0}}$ is the vacuum impedance.

For a large group of $2 \mathrm{D}$ materials in the interband transition regime ${ }^{46,47}$, the $2 \mathrm{D}$ conductivity, $\sigma$ is related to the fine structure constant $\alpha \equiv \frac{e^{2}}{4 \hbar} Z_{0} / \pi \approx 1 / 137$. Graphene ${ }^{46}$, for example, has $\sigma=\frac{\pi \alpha}{Z_{0}}$, whereas semiconductor nanomembranes ${ }^{47}$ have $\sigma=M \cdot \frac{\pi \alpha}{Z_{0}}$, where $M$, an integer, is the number of allowed transitions at a given transition energy. In either case, at normal incidence $\theta=0$, from Equations (7) and (8), we have $\frac{\gamma_{i}}{\gamma_{e}}=\operatorname{Re}(\sigma) Z_{0}=M \cdot \alpha \pi<<1$, where $M=1$ for graphene. Hence, critical coupling cannot be reached at normal incidence, owing to the smallness of the fine structure constant. For intraband transition, the form of $\sigma$ is more complicated. Nevertheless, the condition $\operatorname{Re}(\sigma) Z_{0}<<1$ is still satisfied, and hence one also cannot reach critical coupling in this geometry at $\theta=0$.

However, notice that $\gamma_{i}$ is independent of $\theta$, whereas $\gamma_{e}$ reaches zero at $\theta=\pi / 2$. Therefore, there is always a critical angle of incidence $\theta_{c}$, at which critical coupling is satisfied. 
In the cases considered here, where $\operatorname{Re}(\sigma) Z_{0}<<1$, the critical angle can be determined as:

$$
\theta_{c}=\frac{\pi}{2}-\frac{\varepsilon-1}{\varepsilon} \operatorname{Re}(\sigma) Z_{0}
$$

and is close to $90^{\circ}$. For graphene or semiconductor nanomembranes in the interband transition regime,

$$
\theta_{c}=\frac{\pi}{2}-\frac{\varepsilon-1}{\varepsilon} M \pi \alpha
$$

where $M=1$ for graphene. Therefore, we see that the critical angle is directly related to the fine structure constant in this regime.

As an example, in Figure 1, we consider a single layer of graphene atop a structure consisting of a $1.9-\mu \mathrm{m}$-thick dielectric layer with a refractive index of 2.1 atop a perfect electric conductor layer. The graphene has a Fermi energy of $-500 \mathrm{meV}$ and a mobility of $750 \mathrm{~cm}^{2} \mathrm{~V}^{-1} \mathrm{~s}^{-1}$. The structure supports a resonance in the midinfrared wavelengths. Figure $1 \mathrm{~b}$ shows the external and internal decay rates of the structure. At normal incidence, we indeed see $\gamma_{e}(0)>>\gamma_{i}(0)$. As the angle of incidence increases, Figure 1c shows that critical coupling is achieved at $\theta_{c} \approx 88.4^{\circ}$, where the structure transitions from over-coupling to under-coupling (Figure 1b). The angular dependence of the peak absorptivity is shown in Figure 1d. Around the critical angle, the peak absorptivity is sensitive to the angle of incidence.

We now experimentally demonstrate our concept by showing large angle-selective enhancement of mid-infrared light absorption in single-layer graphene. In our experiment, we place a single layer of chemically doped graphene atop a structure consisting of a $1.9-\mu \mathrm{m}-$ thick $\mathrm{Ge}_{23} \mathrm{Sb}_{7} \mathrm{~S}_{70}$ chalcogenide glass $(\mathrm{ChG})$ layer backed by a gold reflector, as shown in Figure 2a. Here, $\mathrm{Ge}_{23} \mathrm{Sb}_{7} \mathrm{~S}_{70} \mathrm{ChG}$ has relatively weak absorption at mid-infrared wavelengths. It also has a low refractive index ${ }^{48-50}$ of $\sim 2.1$, which is important for reducing the absorption loss inside the metal reflector.

From Equation (9), we see that the same structure can provide complete absorption for different 2D materials with different 2D conductivity $\sigma$. To demonstrate this, we use two different graphene layers with different doping levels. We chemically dope graphene by applying nitric acid vapor and achieve two doping levels by doping graphene for $5 \mathrm{~min}$ and $40 \mathrm{~s}$, respectively. Figure $2 \mathrm{~b}$ shows the Raman spectra of the graphene for the two cases. The single symmetric 2D peak in the Raman spectra confirms that it is single-layer graphene ${ }^{51}$. For different doping, the $G$ and 2D peaks of the Raman spectra shift. Using the experimentally determined relation between the doping level and the positions of $G$ and 2D peaks from Ref. 41, the Fermi energy $E_{F}$ for the graphene with 5-min and 40-s doping are estimated to be $-500 \mathrm{meV}$ and $-300 \mathrm{meV}$, respectively (see the Supplementary Information).

The measured angle-resolved absorptivity for the structures with and without graphene are shown in Figure 2c-2e. We consider $s$-polarization. In Figure $2 c$, for the graphene with $E_{F}=-500 \mathrm{meV}$, the peak absorptivity increases with increasing angle of incidence and reaches $77.6 \%$ at an $89^{\circ}$ angle of incidence. For the graphene with $E_{F}=-300 \mathrm{meV}$ (Figure 2d), the peak absorptivity reaches $69 \%$ at an $89^{\circ}$ angle of incidence. In contrast, the bare structure without graphene exhibits much smaller absorptivity (Figure 2e), and the peak absorptivity is only $43.1 \%$ at an $89^{\circ}$ angle of incidence. The absorption in the bare structure results from parasitic loss inside the gold reflector and the ChG layer. We see that the same structure can be used to enhance the absorption for two different graphene layers at different doping levels.
Figure $2 \mathrm{f}$ shows the absorption in graphene (squares), the parasitic absorption (triangles) and the total absorptivity (diamonds). The absorption in graphene and the parasitic absorption are obtained from experimental results using coupled mode theory formulas. We note that the absorption in graphene is not simply equal to the difference between the peak absorptivities for the structures with and without graphene. For both cases, the absorption in graphene dominates the parasitic absorption. For the structure with the graphene at $E_{F}=-500 \mathrm{meV}$, at an $89^{\circ}$ angle of incidence, the absorption in graphene is $47.2 \%$, and parasitic absorption is $30.4 \%$. For the structure with the graphene at $E_{F}=-300 \mathrm{meV}$, at an $89^{\circ}$ angle of incidence, the absorption in graphene and parasitic absorption are $35.1 \%$ and $34 \%$, respectively. Figure $2 \mathrm{f}$ also shows that as the doping level of graphene increases, the absorption in graphene increases while the parasitic absorption decreases, which is consistent with the coupled mode theory (see Equations (3) and (4)).

The demonstrated $47.2 \%$ light absorption in the single-layer graphene is a significant enhancement of mid-infrared light absorption in single-layer graphene. Previously, the highest experimentally demonstrated mid-infrared light absorption in graphene was 30\% from Ref. 16, by nanopatterning a graphene layer into a graphene nanodisk array. Our achieved absorption enhancement is substantially higher than previously experimentally demonstrated mid-infrared light absorption in single-layer graphene $\mathrm{e}^{15,16,52-54}$ yet is realized using a simpler structure. Our scheme is also compatible with chemical and biological sensing applications ${ }^{17}$, in which the molecules must have contact with the 2D material.

Using an experimentally characterized complex refractive index of $\mathrm{Ge}_{23} \mathrm{Sb}_{7} \mathrm{~S}_{70}$ and tabulated optical constants of $\mathrm{Au}^{55}$, numerical calculation shows that the structure with $E_{F}=-500 \mathrm{meV}$ graphene can achieve near-complete absorption, in which light is predominantly absorbed by graphene (see the Supplementary Information). This calculated absorptivity is higher than the measured result in Figure 2. The difference between the two can be well explained by considering a typical angular spread of the probing light in the measurement system (see the details in the Supplementary Information). Therefore, we expect that our fabricated device may already achieve critical coupling, in which the majority of absorption is in the graphene.

Our approach has the significant advantage that the same structure can provide critical coupling for different $2 \mathrm{D}$ materials with different properties. The specific material properties of the $2 \mathrm{D}$ materials often cannot be precisely controlled in an experiment and are rather characterized only after the device is fabricated. This presents an experimental challenge for other resonant enhancement approaches to enhance light absorption in $2 \mathrm{D}$ materials, owing to the stringent requirement for the critical coupling to achieve perfect resonant absorption. In contrast, our approach provides a simple enhancement approach that can work with $2 \mathrm{D}$ materials as prepared. As an example, in the case of graphene considered here, the conductivity $\sigma_{G}$ of graphene due to intraband transition depends on doping level and mobility. Regardless of these properties of graphene, there is always an angle of incidence, at which critical coupling is met. In Figure 3a, we show the critical angle as a function of Fermi level and mobility. Here, the structure includes a $1.9-\mu \mathrm{m}$-thick spacer layer with a refractive index of 2.1.

The critical angle depends on the conductivity of the 2D material and is not necessarily large. Figure $3 \mathrm{~b}$ shows the critical angle for different doping levels and resonance wavelengths, with the mobility of graphene of $750 \mathrm{~cm}^{2} \mathrm{~V}^{-1} \mathrm{~s}^{-1}$. We observe that the critical angle can be as small as $30^{\circ}$ at far infrared, owing to increasing conductivity 
a

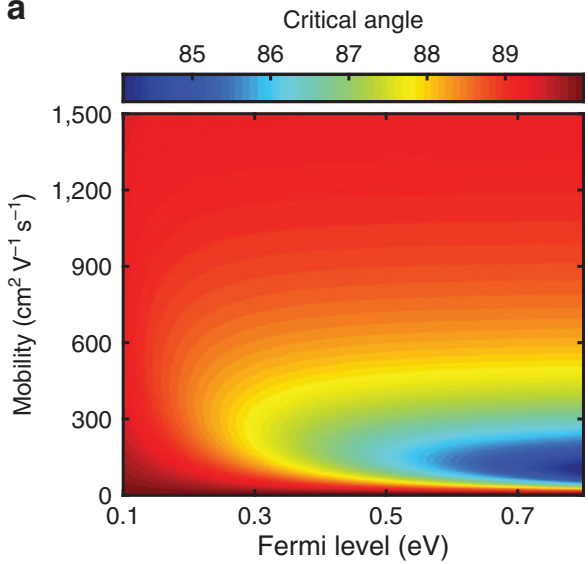

b

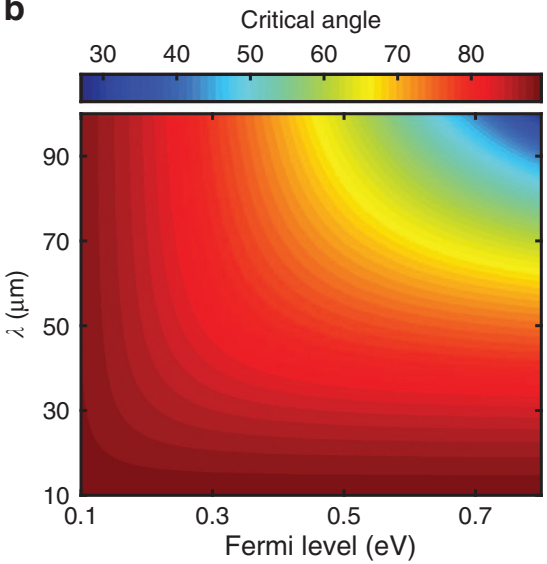

Figure 3 Calculated critical angle for different graphene properties and wavelengths. (a) Calculated critical angle as a function of graphene Fermi level and mobility. The structure consists of a graphene layer on top, a 1.9- $\mu \mathrm{m}$-thick dielectric layer with a refractive index of 2.1 in the middle and a PEC layer on the back. (b) Calculated critical angle as a function of graphene Fermi level and resonance wavelengths. The structure consists of a graphene layer on top, a dielectric layer with a refractive index of 2.1 in the middle and a PEC layer on the back. The graphene has a mobility of $750 \mathrm{~cm}^{2} \mathrm{~V}-1 \mathrm{~s}^{-1}$. In a and $\mathbf{b}$, s-polarization is considered. PEC, perfect electric conductor.

of graphene at longer wavelengths. In addition, for quasi-2D thin films with tuned thickness, the critical angle can be quite small.

Although we discuss the s-polarized case above, angle-selective perfect absorption in a $2 \mathrm{D}$ material can be generalized to $p$-polarization in a geometry consisting of a $2 \mathrm{D}$ material separated from a perfect magnetic conductor mirror ${ }^{56,57}$ by a lossless spacer. In this case, the external and internal decay rates are $\frac{c}{d} \cdot \frac{\varepsilon \cdot \cos \theta}{\varepsilon \mu-\sin ^{2} \theta}$ and $\frac{c}{d} \cdot \frac{\sin ^{2} \theta}{\varepsilon^{2} \mu} \cdot \operatorname{Re}\left(\sigma_{\perp}\right) Z_{0}$, respectively (see the Supplementary Information for derivation). Here, $\sigma_{\perp}$ is the $2 \mathrm{D}$ conductivity of the $2 \mathrm{D}$ material in the vertical direction, and the spacer material has a relative permittivity $\varepsilon$ and relative permeability $\mu$. For small 2D conductivity, the critical angle in $p$-polarization can be described as $\frac{\pi}{2}-\frac{\varepsilon \mu-1}{\varepsilon^{3} \mu} \operatorname{Re}\left(\sigma_{\perp}\right) Z_{0}$ (see the Supplementary Information).

\section{CONCLUSIONS}

In conclusion, we report a general and robust mechanism for achieving angle-selective perfect light absorption in 2D material by placing it on a dielectric spacer layer backed by a mirror. In this geometry, for any standard 2D material in the wavelength range, in which the material experiences loss, we theoretically prove that there always exists an angle of incidence, at which light is completely absorbed in the 2D material. Experimentally, we show high light absorption in single-layer graphene by placing it on a $\mathrm{Ge}_{23} \mathrm{Sb}_{7} \mathrm{~S}_{70}$ chalcogenide layer backed by a mirror, achieving a total absorption of $77.6 \%$ at a wavelength of $\sim 13 \mu \mathrm{m}$, in which the graphene contributes a record-high $47.2 \%$ absorptivity in the mid-infrared wavelength range. Our approach also has the significant advantage that the same structure can provide angle-selective perfect absorption for different $2 \mathrm{D}$ materials with different properties, and we experimentally show angle-selective absorption enhancement on the same structure using graphene with different doping levels. Because our approach does not involve the nanopatterning process, it is robust and can be scaled to large areas with a high yield. Our approach provides a robust platform, on which to achieve angle-selective perfect absorption in 2D materials. Construction of such an angle-selective thin optical element is important for applications ranging from solar and thermal energy harvesting and photo-detection, to chemical and biological sensing. Our results open new avenues for combining 2D materials with photonic structures to enable novel device applications.

\section{AUTHOR CONTRIBUTIONS}

LZ, ZY, XW and SF envisioned and implemented the experimental studies and wrote the manuscript. LZ performed the theoretical analysis, FTIR measurement and numerical analysis. $\mathrm{HL}, \mathrm{JH}, \mathrm{LZ}$ and $\mathrm{ZY}$ deposited the chalcogenide and gold layers. FL and XW deposited, transferred and chemically doped the graphene layers. LZ, FL and XW performed Raman spectroscopy of the samples. All authors discussed the data and commented on the manuscript. ZY, XW and SF supervised the project.

\section{CONFLICT OF INTEREST}

The authors declare no conflict of interest.

\section{ACKNOWLEDGEMENTS}

This work was performed in part at the Stanford Nanofabrication Facility, which is supported by the National Science Foundation through the National Nanotechnology Infrastructure Network (NNIN) under grant number ECS-9731293, and the Stanford Nano Center (SNC), part of the Stanford Nano Shared Facilities. The work at Stanford University is supported by an AFOSR MURI project (FA9550-12-1-0024). The work at Nanjing University is supported by the National Key Basic Research Program of China 2013CBA01604 and 2015CB921600; National Natural Science Foundation of China 61325020, 61261160499 and 11274154. The authors thank Dr Richardson's group at CREOL, UCF for providing the bulk $\mathrm{Ge}_{23} \mathrm{Sb}_{7} \mathrm{~S}_{70}$ glass samples for thin film deposition.

1 Bonaccorso F, Sun Z, Hasan T, Ferrari AC. Graphene photonics and optoelectronics. Nat Photonics 2010; 4: 611-622.

2 Vakil A, Engheta N. Transformation optics using graphene. Science 2011; 332: 1291-1294.

3 Grigorenko AN, Polini M, Novoselov KS. Graphene plasmonics. Nat Photonics 2012; 6: 749-758.

4 de Abajo FJG. Graphene nanophotonics. Science 2013; 339: 917-918.

5 Xia FN, Wang H, Xiao D, Dubey M, Ramasubramaniam A. Two-dimensional material nanophotonics. Nat Photonics 2014; 8: 899-907.

6 Liu M, Yin XB, Ulin-Avila E, Geng BS, Zentgraf T et al. A graphene-based broadband optical modulator. Nature 2011; 474: 64-67.

7 Liu M, Yin XB, Zhang X. Double-Layer graphene optical modulator. Nano Lett 2012; 12: $1482-1485$.

8 Pospischil A, Humer M, Furchi MM, Bachmann D, Guider R et al. CMOS-compatible graphene photodetector covering all optical communication bands. Nat Photonics 2013; 7: 892-896.

9 Gan X, Shiue RJ, Gao Y, Meric I, Heinz TF et al. Chip-integrated ultrafast graphene photodetector with high responsivity. Nat Photonics 2013; 7: 883-887. 
10 Wang XM, Cheng ZZ, Xu K, Tsang HK, Xu JB. High-responsivity graphene/siliconheterostructure waveguide photodetectors. Nat Photonics 2013; 7: 888-891.

11 Youngblood N, Anugrah Y, Ma R, Koester SJ, Li M. Multifunctional graphene optical modulator and photodetector integrated on silicon waveguides. Nano Lett 2014; 14: 2741-2746.

12 Qiu CY, Gao WL, Vajtai R, Ajayan PM, Kono J et al. Efficient Modulation of $1.55 \mu \mathrm{m}$ radiation with gated graphene on a silicon microring resonator. Nano Lett 2014; 14: 6811-6815.

13 Phare CT, Daniel Lee YH, Cardenas J, Lipson M. Graphene electro-optic modulator with $30 \mathrm{GHz}$ bandwidth. Nat Photonics 2015; 9: 511-514.

14 Thongrattanasiri S, Koppens FHL, de Abajo FJG. Complete optical absorption in periodically patterned graphene. Phys Rev Lett 2012; 108: 047401.

15 Fang ZY, Thongrattanasiri S, Schlather A, Liu Z, Ma LL et al. Gated tunability and hybridization of localized plasmons in nanostructured graphene. ACS Nano 2013; 7: 2388-2395.

16 Fang ZY, Wang YM, Schlather AE, Liu Z, Ajayan PM et al. Active tunable absorption enhancement with graphene nanodisk arrays. Nano Lett 2014; 14: 299-304.

17 Rodrigo D, Limaj O, Janner D, Etezadi D, de Abajo FJG et al. Mid-infrared plasmonic biosensing with graphene. Science 2015; 349: 165-168.

18 Gao WL, Shu J, Quu CY, Xu QF. Excitation of plasmonic waves in graphene by guidedmode resonances. ACS Nano 2012; 6: 7806-7813.

19 Zhan TR, Zhao FY, Hu XH, Liu XH, Zi J. Band structure of plasmons and optical absorption enhancement in graphene on subwavelength dielectric gratings at infrared frequencies. Phys Rev B 2012; 86: 165416.

20 Majumdar A, Kim J, Vuckovic J, Wang F. Electrical control of silicon photonic crystal cavity by graphene. Nano Lett 2013; 13: 515-518.

21 Wu SF, Buckley S, Jones AM, Ross JS, Ghimire NJ et al. Control of two-dimensional excitonic light emission via photonic crystal. 2D Mater 2014; 1: 011001.

22 Piper JR, Fan SH. Total absorption in a graphene monolayer in the optical regime by critical coupling with a photonic crystal guided resonance. ACS Photonics 2014; 1: 347-353.

23 Liu YH, Chadha A, Zhao DY, Piper JR, Jia YC et al. Approaching total absorption at near infrared in a large area monolayer graphene by critical coupling. Appl Phys Lett 2014; 105: 181105.

24 Wu SF, Buckley S, Schaibley JR, Feng LF, Yan JQ et al. Monolayer semiconductor nanocavity lasers with ultralow thresholds. Nature 2015; 520: 69-72.

25 Gao YD, Shiue RJ, Gan XT, Li LZ, Peng C et al. High-speed electro-optic modulator integrated with graphene-boron nitride heterostructure and photonic crystal nanocavity. Nano Lett 2015; 15: 2001-2005.

26 Lin HT, Sun XC, Liu JF, Hu JJ. Diffractive broadband coupling into high-Q resonant cavities. Opt Lett 2015; 40: 2377-2380.

27 Wang WY, Klots A, Yang YM, Li W, Kravchenko II et al. Enhanced absorption in two-dimensional materials via Fano-resonant photonic crystals. Appl Phys Lett 2015; 106: 181104

28 Xia FN, Mueller T, Golizadeh-Mojarad R, Freitage M, Lin YM et al. Photocurrent imaging and efficient photon detection in a graphene transistor. Nano Lett 2009; 9: 1039-1044.

29 Xia FN, Mueller T, Lin YM, Valdes-Garcia A, Avouris P. Ultrafast graphene photodetector. Nat Nanotechnol 2009; 4: 839-843.

30 Mueller T, Xia FN, Avouris P. Graphene photodetectors for high-speed optical communications. Nat Photonics 2010; 4: 297-301.

31 Furchi M, Urich A, Pospischil A, Lilley G, Unterrainer K et al. Microcavity-integrated graphene photodetector. Nano Lett 2012; 12: 2773-2777.

32 Cai XH, Sushkov AB, Suess RJ, Jadidi MM, Jenkins GS et al. Sensitive roomtemperature terahertz detection via the photothermoelectric effect in graphene. Nat Nanotechnol 2014; 9: 814-819.

33 Liu CH, Chang YC, Norris TB, Zhong ZH. Graphene photodetectors with ultra-broadband and high responsivity at room temperature. Nat Nanotechnol 2014; 9: 273-278.

34 Liu JT, Liu NH, Li J, Li XJ, Huang JH. Enhanced absorption of graphene with onedimensional photonic crystal. Appl Phys Lett 2012; 101: 052104.

35 Kosten ED, Atwater JH, Parsons J, Polman A, Atwater HA. Highly efficient GaAs solar cells by limiting light emission angle. Light Sci Appl 2013; 2: e45.
36 Raman AP, Anoma MA, Zhu LX, Rephaeli E, Fan SH. Passive radiative cooling below ambient air temperature under direct sunlight. Nature 2014; 515: 540-544.

37 Hamam R, Celanovic I, Soljačić M. Angular photonic band gap. Phys Rev A 2011; 83: 035806-1-035806-4.

38 Shen YC, Ye DX, Celanovic I, Johnson SG, Joannopoulos JD et al. Optical broadband angular selectivity. Science 2014; 343: 1499-1501.

39 Liang XL, Sperling BA, Calizo I, Cheng GJ, Hacker CA et al. Toward clean and crackless transfer of graphene. ACS Nano 2011; 5: 9144-9153.

40 Wang XR, Li XL, Zhang L, Yoon Y, Weber PK et al. N-doping of graphene through electrothermal reactions with ammonia. Science 2009; 324: 768-771.

41 Das A, Pisana S, Chakraborty B, Piscanec S, Saha SK et al. Monitoring dopants by Raman scattering in an electrochemically top-gated graphene transistor. Nat Nanotechnol 2008; 3: 210-215.

42 Haus HA. Waves and Fields in Optoelectronics. Prentice Hall: New Jersey; 1984.

43 Suh W, Wang Z, Fan SH. Temporal coupled-mode theory and the presence of nonorthogonal modes in lossless multimode cavities. IEEE J Quantum Electron 2004; 40: 1511-1518.

44 Horng J, Chen CF, Geng BS, Girit C, Zhang YB et al. Drude conductivity of Dirac fermions in graphene. Phys Rev B 2011; 83: 165113.

45 Novoselov KS, Geim AK, Morozov SV, Jiang D, Katsnelson MI et al. Two-dimensional gas of massless Dirac fermions in graphene. Nature 2005; 438: 197-200.

46 Nair RR, Blake P, Grigorenko AN, Novoselov KS, Booth TJ et al. Fine structure constant defines visual transparency of graphene. Science 2008; 320: 1308.

47 Fang $\mathrm{H}$, Bechtel HA, Plis E, Martin MC, Krishna S et al. Quantum of optical absorption in two-dimensional semiconductors. Proc Natl Acad Sci USA 2013; 110: 11688-11691.

48 Musgraves JD, Carlie N, Hu J, Petit L, Agarwal A et al. Comparison of the optical, thermal and structural properties of Ge-Sb-S thin films deposited using thermal evaporation and pulsed laser deposition techniques. Acta Mater 2011; 59: 5032-5039.

49 Lin HT, Li L, Zou Y, Danto S, Musgraves JD et al. Demonstration of high-Q mid-infrared chalcogenide glass-on-silicon resonators. Opt Lett 2013; 38: 1470-1472.

$50 \mathrm{Li}$ L, Lin HT, Qiao ST, Zou Y, Danto S et al. Integrated flexible chalcogenide glass photonic devices. Nat Photonics 2014; 8: 643-649.

51 Ferrari AC, Basko DM. Raman spectroscopy as a versatile tool for studying the properties of graphene. Nat Nanotechnol 2013; 8: 235-246.

52 Yan HG, Low T, Zhu WJ, Wu YQ, Freitag M et al. Damping pathways of mid-infrared plasmons in graphene nanostructures. Nat Photonics 2013; 7: 394-399.

53 Freitag M, Low T, Zhu WJ, Yan HG, Xia FN et al. Photocurrent in graphene harnessed by tunable intrinsic plasmons. Nat Commun 2013; 4: 1951.

54 Zhu XL, Yan W, Jepsen PU, Hansen O, Mortensen NA et al. Experimental observation of plasmons in a graphene monolayer resting on a two-dimensional subwavelength silicon grating. App/ Phys Lett 2013; 102: 131101.

55 Johnson PB, Christy RW. Optical constants of the noble metals. Phys Rev B 1972; 6 : 4370-4379.

56 Sievenpiper D, Zhang LJ, Broas RFJ, Alexópolous NG, Yablonovitch E. High-impedance electromagnetic surfaces with a forbidden frequency band. IEEE Trans Microw Theory Tech 1999; 47: 2059-2074.

57 Esfandyarpour M, Garnett EC, Cui Y, McGehee MD, Brongersma ML. Metamaterial mirrors in optoelectronic devices. Nat Nanotechnol 2014; 9: 542-547.

This work is licensed under a Creative Commons Attribution(c) $(1)$ ()
other third party material in this article are included in the article's Creative Commons other third party material in this article are included in the article's Creative Commons the Creative Commons license, users will need to obtain permission from the license holder to reproduce the material. To view a copy of this license, visit http:// creativecommons.org/licenses/by-nc-sa/4.0/

Supplementary Information for this article can be found on the Light: Science \& Applications' website (http://www.nature.com/lsa) 